Y. C. Minh and E. F. van Dishoeck, eds.

\title{
Models of Gas-Grain Chemistry in Star-forming Regions
}

\author{
Eric Herbst \\ Departments of Physics and Astronomy, The Ohio State University, \\ Columbus, OH 43210, USA
}

\begin{abstract}
It is difficult if not impossible to explain the abundances of assorted interstellar molecules in both the gaseous and condensed phases without the use of grain chemistry. Unfortunately, the chemistry occurring on grains is not well understood because of a variety of uncertainties including the nature, size, and shape of dust particles, the binding energies of key species, the dominant mechanism of surface chemistry, and the correct mathematical treatment of surface processes. Still, intrepid astrochemists have used granular chemistry in chemical models of an assortment of sources including cold clouds, protostellar disks, and hot cores. Indeed, the dominant explanation of the saturated gas-phase molecules observed in hot cores involves grain chemistry during an earlier, low temperature phase. Although gas-grain models have elucidated major features of the chemistry, much more work remains to be accomplished before they can be used to help characterize the physical conditions in star-forming regions and their temporal variations.
\end{abstract}

\section{The Need for Grain Chemistry}

Of the more than 100 different gas-phase molecules detected via high resolution spectroscopy in interstellar and circumstellar clouds, gas-phase chemistry can qualitatively or even quantitatively explain the abundances of many but not all of them (e.g. Terzieva \& Herbst 1998). The simplest molecule which requires chemistry on grain surfaces for its formation is molecular hydrogen, which is the most abundant molecule in interstellar clouds by a factor of $10^{4}$ ! Another diatomic molecule for which gas-phase processes do not provide a sufficient explanation, at least in diffuse clouds, is $\mathrm{NH}$ (Wagenblast et al. 1993). A third relatively simple molecule, methanol $\left(\mathrm{CH}_{3} \mathrm{OH}\right)$, can possibly be produced in the gas via the radiative association between $\mathrm{CH}_{3}^{+}$and $\mathrm{H}_{2} \mathrm{O}$ :

$$
\mathrm{CH}_{3}^{+}+\mathrm{H}_{2} \mathrm{O} \longrightarrow \mathrm{CH}_{3} \mathrm{OH}_{2}^{+}+\mathrm{h} \nu,
$$

followed by dissociation recombination with electrons to form $\mathrm{CH}_{3} \mathrm{OH}+\mathrm{H}$. Neither reaction has been studied in the laboratory, and the estimates for the rate of the radiative association may be insufficient to explain even the small abundance of methanol measured in the gas of dark and translucent clouds (Turner 1998). The hydrogenation of CO into methanol on grain surfaces via successive reactions with $\mathrm{H}$ atoms, studied in the laboratory by Hiraoka et al. $(1994,1998)$, is at least as likely a mechanism for its formation in interstellar space, although 
in cold regions the desorption of the product back into the gas phase is still poorly understood. This hydrogenation requires four reactions, two of which possess known but uncertain activation energy:

$$
\begin{gathered}
\mathrm{H}+\mathrm{CO} \longrightarrow \mathrm{HCO}, \\
\mathrm{H}+\mathrm{H}_{2} \mathrm{CO} \longrightarrow \mathrm{H}_{3} \mathrm{CO},
\end{gathered}
$$

but, unlike the gas phase, activation energies do not necessarily prohibit reactions from occurring on grain surfaces since tunneling can occur with high efficiency (Tielens \& Hagen 1982).

The case for a granular origin for gas-phase species is especially strong in hot cores, which are dense, warm but quiescent regions $\left(n \approx 10^{6}-10^{7} \mathrm{~cm}^{-3}\right.$; $T \approx 100-300 \mathrm{~K}$ ) associated with high mass star-forming regions. In Orion, two such regions are known as the Hot Core and the Compact Ridge (Wright et al. 1992), while in Sgr B2, Snyder, Kuan, \& Miao (1994) have referred to a northern hot core as the "Large Molecule Heimat." In all of these objects, there are a large number of saturated (H-rich) gas-phase species with surprisingly high abundances. Among smaller species, water, ammonia, and $\mathrm{H}_{2} \mathrm{~S}$ are especially plentiful. As is the case for water and ammonia, the abundance of methanol can increase by $2-3$ orders of magnitude over its normal dense cloud value. In addition to methanol, other oxygen-containing organic molecules with high abundances include methyl formate $\left(\mathrm{HCOOCH}_{3}\right)$, methyl ether $\left(\mathrm{CH}_{3} \mathrm{OCH}_{3}\right)$, ethanol $\left(\mathrm{C}_{2} \mathrm{H}_{5} \mathrm{OH}\right)$, and ethylene glycol (cyclic- $\left.\mathrm{CH}_{2} \mathrm{OCH}_{2}\right)$. Nitrogen-containing organic molecules such as vinyl cyanide $\left(\mathrm{C}_{2} \mathrm{H}_{3} \mathrm{CN}\right)$, and cyanoethane $\left(\mathrm{C}_{2} \mathrm{H}_{5} \mathrm{CN}\right)$ are also abundant. There is some evidence in Orion that the oxgyen-containing and nitrogen-containing organic molecules are at least partially segregated (Blake et al. 1987; Irvine, Goldsmith, \& Hjalmarson 1987).

The copious amounts of water detected in hot cores can be produced on cold grains via successive hydrogenation of oxygen atoms by hydrogen atoms during a previous colder era followed by evaporation as the temperature rises, although it is also possible that shock chemistry can enhance the water abundance (Bergin, Neufeld, \& Melnick 1999). Similar hydrogenation of nitrogen and sulphur atoms leads to large abundances of ammonia and $\mathrm{H}_{2} \mathrm{~S}$ respectively. While the detailed formation mechanisms of most of the saturated organic species are still uncertain, it is likely that they are formed at least partially via gas-phase reactions from precursors such as methanol (Millar, Herbst, \& Charnley 1991), which is produced in high abundance on grain surfaces during a previous colder era via the hydrogenation of $\mathrm{CO}$. Another possibility is that these more complex species are themselves synthesized on cold grains (Charnley 1997).

There are two types of gas-grain chemical models of hot cores. In one, which can be labelled the "last refuge of a scoundrel school," the grain chemistry is not treated at all given the many uncertainties (Charnley, Tielens, \& Millar 1992). Rather, specific abundances of precursor molecules are assumed to desorb from grains and the subsequent gas-phase chemistry is followed closely (Charnley et al. 1995; Viti \& Williams 1999). The desorbing molecules and their abundances are chosen based on chemical reasonableness and on the need for the models to reproduce observations. The second type of model actually follows both the gas and grain chemistries during the cold era prior to the era of star formation. Examples include the initial hot core models of the UMIST group (Brown, 
Charnley, \& Millar 1988; Brown \& Millar 1989a,b) as well as the more recent work of Caselli, Hasegawa, \& Herbst (1993), in which specific chemical differences between the Hot Core and Compact Ridge in Orion were found to derive from different temperatures and densities in the prior era. A still more detailed model, by Millar \& Hatchell (1998), divides hot core sources into several zones.

Besides the gas-phase molecules that must be accounted for at least partially via grain chemistry, there are molecules detected on grain surfaces in sufficiently high abundance that they cannot be explained via gas-phase syntheses followed by condensation. Perhaps the best example is water ice, which is detected along many lines of sight to have an equivalent fractional abundance with respect to $n$ of $\approx 10^{-4}$. Such a high abundance can be accounted for by grain chemistry (e.g. Hasegawa, Herbst, \& Leung 1992) while low-temperature gas-phase models must struggle to produce abundances of water that are three orders of magnitude lower (Lee, Bettens, \& Herbst 1996). Sizeable amounts of surface carbon dioxide $\left(\mathrm{CO}_{2}\right)$ have also been observed although the synthesis of this species on grains is uncertain due to our lack of knowledge concerning the activation energy of the process

$$
\mathrm{CO}+\mathrm{O} \longrightarrow \mathrm{CO}_{2}
$$

\section{Granular Processes and Models}

Consider the surface of an interstellar grain that is either bare or covered with monolayers of material. The often stated size of a "typical" grain is $\approx 0.10 \mu \mathrm{m}$, but there is a wide distribution of sizes. Adsorption of neutral material striking such grains occurs with high probability $(0.3-1.0)$ at the low temperatures of cold interstellar clouds (Williams 1993). The fate of ionic species is less clear (Aikawa \& Herbst 1999) and may depend on the grain charge. Some processes that can occur to a gas-phase species after it accretes onto a dust particle and bonds weakly to the surface at a site of lowered potential energy are shown in Figure 1. Upon landing on the grain and becoming thermalized, the particle need not be stationary but can travel to other of the $\approx 10^{6}$ binding sites by either hopping over the barriers between sites or actually tunneling beneath them. The size of the barrier against diffusion $\left(E_{\mathrm{b}}\right)$ is typically a fraction of the energy needed for evaporation $\left(E_{\mathrm{D}}\right)$. The mathematics of diffusion is summarized in Tielens \& Hagen (1982), Tielens \& Allamandola (1987), and Hasegawa et al. (1992). Although shape is not considered in these simple treatments, diffusion on irregular grains is likely to be less regular than on relatively flat surfaces. If the diffusing particle finds another species at the same site, reaction can occur if the activation energy is small or zero. A chemistry based on diffusion of this sort is known as a Langmuir-Hinshelwood chemistry. Such a mechanism occurs when there are few reactive species on a surface and when these reactive species can diffuse readily. With standard assumptions concerning diffusion rates on interstellar grains, a Langmuir-Hinshelwood chemistry occurs for the lighter and more reactive surface species such as the atoms $\mathrm{H}, \mathrm{O}, \mathrm{C}, \mathrm{N}$ and light radicals (Tielens \& Hagen 1982; d'Hendecourt, Allamandola, \& Greenberg 1985; Hasegawa et al. 1992). Indeed, the diffusion rate of $\mathrm{H}$ is thought to be much faster than that of any other reactive species so that hydrogenation is an important process. Definitive values for the diffusion rates of various reactive 


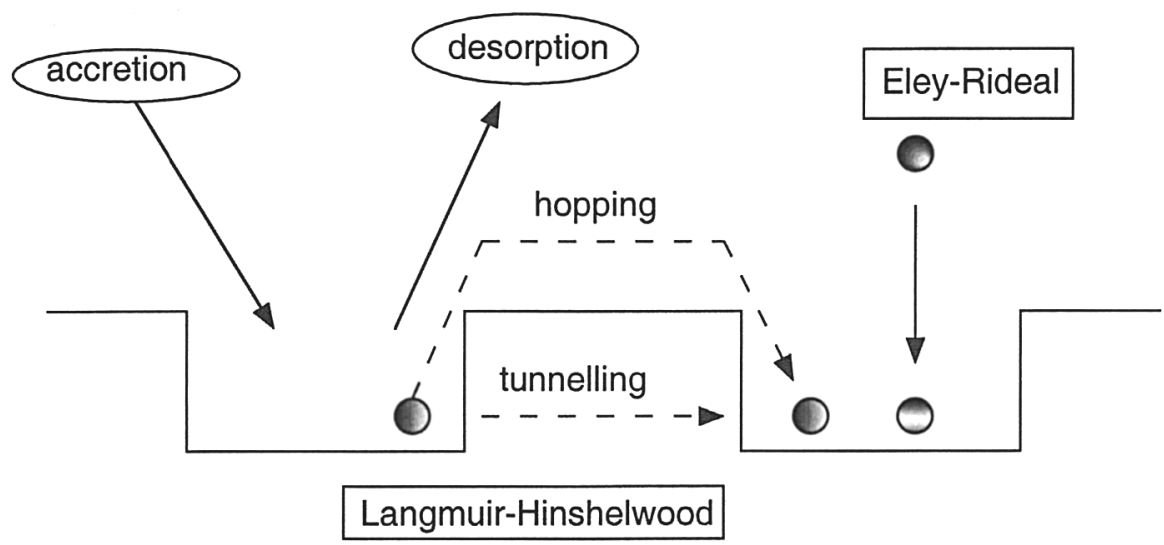

Figure 1. Assorted processes occurring on interstellar grain mantles.

interstellar surface species have been slow in coming although we now have detailed information concerning atomic $\mathrm{H}$ (Katz et al. 1999). It is assumed that for the most part, diffusive grain reactions are associative in nature; the two reacting species combine to form one product with the extra chemical energy mainly warming the grain or being used in some other manner. The evidence is sparse, however, on the branching fractions between associative (single) and regular (multiple) products (see Hiraoka et al. 1998 for some information on the subject).

If the accreted species are strongly bound and cannot move, then another mechanism for surface reactions - the Eley-Rideal mechanism - dominates. In this process, a gas-phase species lands atop or near a stationary reactive species and reaction ensues. Although there are no interstellar models that utilize the Eley-Rideal mechanism, this type of chemistry may well occur preferentially on very small grains where surface species are more likely to be strongly bound and therefore stationary because the weakly-bound species are less likely to stick and are susceptible to evaporation following transient events that raise the temperature (Tielens 1993).

In addition to evaporation, which is very slow at cold interstellar temperatures for all species other than helium, atomic, and molecular hydrogen, there are a variety of non-thermal desorption mechanisms that have been discussed over the years with varying degrees of understanding (Williams 1993,1998). These mechanisms include sputtering in shocks, sputtering and evaporation following cosmic ray bombardment (especially by the more heavy nuclei), use of the energy generated by chemical reactions, photodesorption via high energy (UV) photons or even low energy (IR) photons, grain-grain collisions, grain explosions, etc. Chemical reactions such as the formation of $\mathrm{H}_{2}$ from two $\mathrm{H}$ atoms can cause direct desorption of the product and can heat up the immediate surface of a normal grain to induce the desorption of non-reactive but nearby species. According to 
recent experiments on $\mathrm{H}_{2}$ formation by Katz et al. (1999), more than $50 \%$ of the newly formed hydrogen molecules desorb in the process. Recent theoretical work (Takahashi, this volume) on the indirect process appears to indicate that it is not efficient.

Despite the many uncertainties involved in grain chemistry, a large number of intrepid souls have produced chemical models of interstellar clouds in which grain chemistry plays a role. Among early work, the names of Watson \& Salpeter (1972) and Allen \& Robinson (1977) stand out. Perhaps the three most influential papers are those of Pickles \& Williams (1977), Tielens \& Hagen (1982) and d'Hendecourt et al. (1985) in which the diffusive chemistry occurring on cold interstellar grains was first treated in detail by the two methods in use today - the Monte Carlo (stochastic) approach and the rate equation approach. The latter (or a modification discussed below) is used more frequently because it is the more practical. It was utilized in a series of papers on cold interstellar clouds with a dramatically expanded chemistry by Hasegawa et al. (1992), Hasegawa \& Herbst (1993a,b), and Shalabiea, Caselli, \& Herbst (1998). Shalabiea \& Greenberg (1995) used the rate approach to show that the bistable nature of purely gas-phase chemical models of dense interstellar clouds does not survive the inclusion of grain chemistry. The use of rate-based, gas-grain models in hot core chemistry, already discussed above, has been undertaken by Brown et al. (1988), Brown \& Millar (1989a,b), Caselli et al. (1993), and Millar \& Hatchell (1998). Grain chemistry in protoplanetary disks was first done by Willacy et al. (1998) using rate equations. A limited Monte Carlo approach to the hydrogenation and deuteration of CO in hot core-type sources was reported by Charnley, Tielens, \& Rodgers (1997).

The differing models show qualitatively that, if one starts with a purely atomic gas, the granular chemistry at early time is mainly one of hydrogenation to form simple saturated species such as water and ammonia, while at later times the relatively low abundance of atomic hydrogen and the high abundances of gas-phase molecules such as $\mathrm{CO}$ and $\mathrm{O}_{2}$ lead to a more complex grain chemistry in which non-saturated species such as $\mathrm{CO}_{2}$ can be formed competitively. At still later times, in those models with inefficient desorption so that heavy gas-phase molecules tend to be accreted onto grains, a second phase of hydrogenation chemistry can take place. Thus, models tend to lead to striated grains with different chemical environments depending upon the radii of the deposited monolayers, a result seemingly in agreement with observations (Whittet 1993).

\section{Monte Carlo and Rate Equation Methods}

The rate equation approach to Langmuir-Hinshelwood chemistry is based on the equations used to model gas-phase chemistry (Pickles \& Williams 1977). Let $C$ be a surface species that is formed by the association of surface species $A$ and $B$. Let $N_{\mathrm{A}}, N_{\mathrm{B}}$, and $N_{\mathrm{C}}$ represent the number of molecules of species $A, B$, and $C$ on a particular grain. In the rate equation formalism, the rate of formation of species $\mathrm{C}$ is given by the equation:

$$
\frac{d N_{\mathrm{C}}}{d t}=k N_{\mathrm{A}} N_{\mathrm{B}}
$$


where the surface rate coefficient $k\left(\mathrm{~s}^{-1}\right)$ is the sum of the diffusion or "sweeping" rates of species $A$ and $B$ over the entire surface of the grain. If, instead of unitless numbers of molecules per grain, it is preferred to use concentrations per unit volume, the rate coefficients have the same units $\left(\mathrm{cm}^{3} \mathrm{~s}^{-1}\right)$ as do their gasphase counterparts (Hasegawa et al. 1992). In a gas-grain model, one generally includes adsorption and desorption terms in both the rate equations for gaseous and surface species.

The rate equation approach is perfectly justified in the case in which large numbers of species are reacting on a surface. Let us call this limit the "reactive limit." In the interstellar case, however, the rate of accretion onto individual dust particles is slow enough that very few reactive species exist on the same grain at the same time. Indeed, unless a surface species (such as CO) is weakly reactive due to activation energy barriers, it is most probable that at most two reactive atoms/molecules co-exist per grain at any time. In this situation, called the "accretion limit" by Tielens, the rates of diffusion of the species are not important as long as they are large enough for the species to find one another and react. Furthermore, there is a finite chance (especially critical for $\mathrm{H}_{2}$ formation) that the first atom will evaporate before the second arrives so that no reaction can occur. The rate equation method, which is similar to a mean field approximation, will always determine an average $\mathrm{H}$ atom abundance per grain and, consequently, a finite rate of molecular hydrogen formation which depends on the rate of diffusion and can, under certain conditions, be much too large (Tielens 1995, unpublished).

In the "accretion" limit, the chemistry is more exactly treated by a Monte Carlo procedure in which gas-phase species are randomly selected to adsorb onto a grain and, once on the grain, react with another species if this is more likely than evaporation, or, in the case that more than one co-reactant is available, react with one or the other according to their relative probabilities. Although the Monte Carlo procedure is clearly closer to the reality of interstellar diffusive chemistry than is the rate equation method, it is difficult to include in a timedependent method when the gas-phase chemistry is handled by rate equations. Indeed, to the best of our knowledge, the Monte Carlo procedure has been used for surface chemistry only when the gas-phase abundances are assumed to be constant (Tielens \& Hagen 1982; Caselli et al. 1998; Charnley et al. 1997) so that, given a particular set of gas-phase abundances, one can see what will develop on the grain within a short period. With the rate equation method, on the other hand, one can truly study time-dependent kinetics simultaneously in the gas and on the grain without an exhorbitant use of computer time. Another possibility is that both the gas and granular chemistries can be treated simultaneously by Monte Carlo (or, more rigorously, stochastic) procedures but this is likely to be very time consuming computationally (Charnley 1998) and may well require parallel architecture.

How inaccurate is the rate equation method? As well as reporting their time-dependent gas-grain models, Hasegawa et al. (1992) attempted to reproduce the results of the time-independent late-cloud Monte Carlo model of Tielens \& Hagen (1992), and showed reasonable agreement between the two approaches. Some time later, however, Tielens (1995, unpublished) announced that in a simple model there are situations in which the two approaches differ markedly. 
Caselli et al. (1998) reproduced what they took to be the spirit of Tielens' remarks. In their simple model, they assumed constant abundances of gas-phase $\mathrm{H}$ and $\mathrm{O}$ atoms at a temperature of $10 \mathrm{~K}$, followed their adsorption onto a grain, considered the following efficient grain surface reactions:

$$
\begin{gathered}
\mathrm{H}+\mathrm{H} \longrightarrow \mathrm{H}_{2}, \\
\mathrm{H}+\mathrm{O} \longrightarrow \mathrm{OH}, \\
\mathrm{O}+\mathrm{O} \longrightarrow \mathrm{O}_{2},
\end{gathered}
$$

and monitored the production of $\mathrm{H}_{2}, \mathrm{O}_{2}$, and $\mathrm{OH}$ on the surface by both rate equations and a Monte Carlo approach. In the interest of simplicity, evaporation and non-thermal desorption of the products were ignored. Although the surface concentrations of all three species continually increase, steady-state values of the mole fractions (fractional abundances on the mantle) of each species are reached quickly in both methods. The two sets of computed mole fractions are only sometimes in agreement. At high $\mathrm{H} / \mathrm{O}$ gas-phase ratios, the dominant surface species is $\mathrm{H}_{2}$, while at low $\mathrm{H} / \mathrm{O}$ gas-phase ratios, the dominant surface species is $\mathrm{O}_{2}$. The radical $\mathrm{OH}$ dominates in a middle range. The transition zones between regions of domination can be quite different, however; the Monte Carlo results generally show that the $\mathrm{O}_{2} / \mathrm{OH}$ and the $\mathrm{OH} / \mathrm{H}_{2}$ boundaries lie at considerably higher values of gas-phase $\mathrm{H} / \mathrm{O}$ than do the rate method results. Since these boundaries occur at abundances for gas-phase $\mathrm{H}$ and $\mathrm{O}$ that are characteristic of older dense clouds, the disagreements are important and troublesome although it is not at all clear that they will persist for larger and more complex reaction networks.

\section{Modified Surface Rates}

In an effort to improve the rate equation approach, Caselli et al. (1998) modified the rate coefficients for the simple $\mathrm{O} / \mathrm{H}$ system at $10 \mathrm{~K}$ discussed above (as well as some slightly more complex systems) and showed that the modifications, which are semi-empirical in nature, lead to quantitative agreement with the Monte Carlo approach. The basic idea of the modifications is to build discreteness into the rate equations. This is done in two ways: (1) by defining a discrete unit of time such that no process can occur within a shorter period, and (2) by allowing for the evaporation of one reactive species (typically $\mathrm{H}$ ) from a grain before a second reactive species can accrete. The unit of time is either the evaporation time of $\mathrm{H}$ (estimated to be $500 \mathrm{~s}$ at $10 \mathrm{~K}$ ) or the interval between accretion of $\mathrm{H}$ atoms (dependent on density), whichever is shorter. It is incorporated into the rate equations by artificially slowing the diffusion rate of atomic $\mathrm{H}$ so that at most one hydrogenation reaction per grain can occur during the quantum of time.

Extensions of the modifications were then put into a complete gas-grain model of cold dense clouds by Shalabiea et al. (1998) and differences noted with the results obtained with unmodified surface rate coefficients. Two cases were studied distinguished by their initial conditions: Case A, in which the hydrogen in the gas is initially in molecular form, and Case B, in which the hydrogen is initially in atomic form. Few differences are expected for Case B 


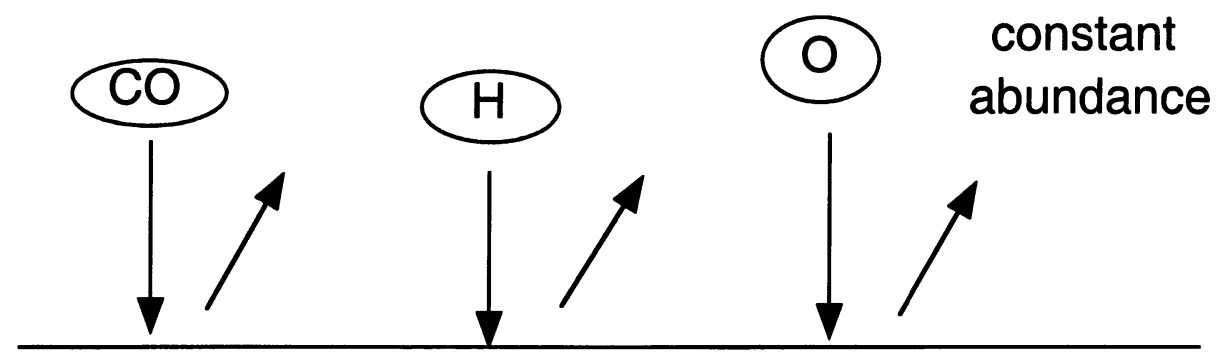

\section{dust particle}

Figure 2. Model for simple comparisons of the Monte Carlo and modified rate approaches.

since hydrogenation will prevail with both unmodified and modified rates, and few differences are found. For Case A, the story is more complex, with some large differences persisting up to $10^{6} \mathrm{yr}$ or even longer. In particular, the surface abundance of ammonia is found to be several orders of magnitude lower with the modified rates, although there is hardly any difference in the results for methanol, another hydrogenation product. Molecular nitrogen, on the other hand, is more abundant through $10^{5} \mathrm{yr}$ with the modified rates. At sufficiently long times, the differences tend to vanish for almost all species.

The modified rates have not been used for hot core models because, to the present, they have only been "derived" for $10 \mathrm{~K}$ grains, and it is necessary to have a more flexible approach able to take into account thermal variations.

In work in progress, Caselli \& Herbst are extending the rate modifications to work in the temperature range $10-20 \mathrm{~K}$ and are looking more carefully at the proper manner in which to modify the rates of reactions with activation energy such as (2) and (3). They are once again testing their semi-empirical approach by comparison with a Monte Carlo method for a system with fixed gas-phase abundances. As shown in Figure 2, three species - $\mathrm{CO}, \mathrm{O}$, and $\mathrm{H}-$ are considered to be in the gas at constant abundance and to accrete onto a grain for approximately $1000 \mathrm{yr}$. Ten surface reactions are included, leading to the production of $\mathrm{H}_{2}, \mathrm{O}_{2}, \mathrm{OH}, \mathrm{H}_{2} \mathrm{O}, \mathrm{HCO}, \mathrm{H}_{2} \mathrm{CO}, \mathrm{H}_{3} \mathrm{CO}, \mathrm{CH}_{3} \mathrm{OH}$ and $\mathrm{CO}_{2}$. The carbon dioxide is produced via the direct reaction between $\mathrm{CO}$ and $\mathrm{O}$ (reaction $[4])$, and by the reaction

$$
\mathrm{HCO}+\mathrm{O} \longrightarrow \mathrm{CO}_{2}+\mathrm{H}
$$

Since reaction (4) is assumed to have an activation energy and since $\mathrm{O}$ and $\mathrm{CO}$ do not move rapidly on surfaces, this latter reaction dominates $\mathrm{CO}_{2}$ production. Reactions (2) and (3) are assumed to have activation energy barriers of $2500 \mathrm{~K}$. Once again, steady-state abundances (in the sense of mole fractions if not actual concentrations) are reached within a short period of time. Figure 3 shows computed mole fractions of the dominant surface species at $1000 \mathrm{yr}$ in the 10-20 K range for the following gas densities (referred to in the figure as "low density"): $n_{\mathrm{H}}=1.15 \mathrm{~cm}^{-3}, n_{\mathrm{O}}=0.09 \mathrm{~cm}^{-3}$ and $n_{\mathrm{CO}}=0.075 \mathrm{~cm}^{-3}$. The concentrations come from steady-state gas-phase model results of Lee et al. (1996) for 


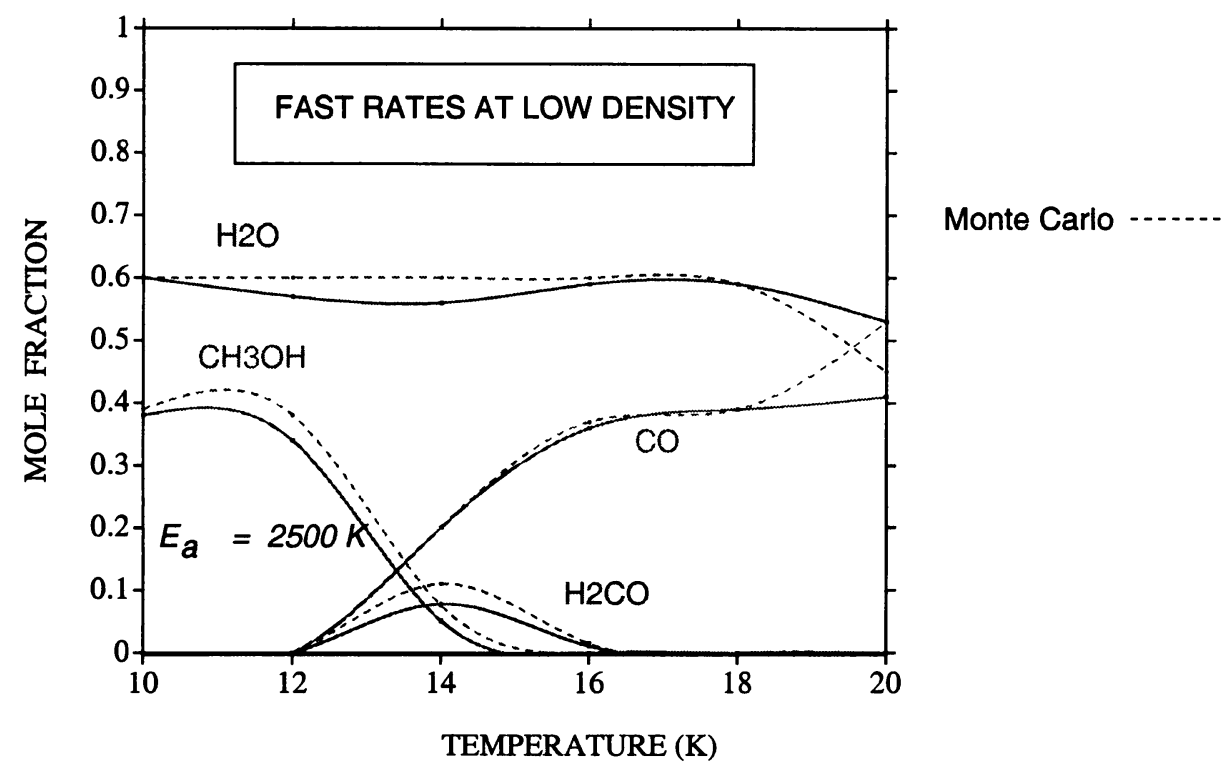

Figure 3. Results of comparison between the Monte Carlo and modified rate approaches at $10-20 \mathrm{~K}$ using so-called "fast" surface rate coefficients at a fixed "low" density.

a molecular hydrogen gas density of $500 \mathrm{~cm}^{-3}$. The designation "fast rates" in the figure does not refer to unmodified rate coefficients but rather to modified rates based on the diffusion barriers listed in Hasegawa et al. (1992). The case of "slow rates" will be discussed below. In the figure, the Monte Carlo results are presented as dashed lines while the modified rate results are presented as solid lines. Very little difference can be seen between the results of the two approaches in this case. Both show that water dominates at most temperatures, and that the hydrogenation of $\mathrm{CO}$ proceeds further at lower temperatures than at higher temperatures. The reason is that evaporation of $\mathrm{H}$ atoms competes with hydrogenation, and at higher temperatures evaporation begins to dominate. The surface molecule $\mathrm{CO}_{2}$ is found to be abundant for gas mixtures somewhat poorer in atomic $\mathrm{H}$.

\subsection{Slow rates?}

The diffusion rates used for our models are quite approximate in the sense that the barriers against diffusion are rarely known precisely and are quite dependent on the nature of the surface anyway. In general, we have followed earlier authors in the assumption that $E_{\mathrm{b}}$, the diffusion barrier, is approximately $0.30 \times E_{\mathrm{D}}$, the barrier against desorption (Tielens \& Allamandola 1987). For atomic $\mathrm{H}$, the numbers used are $100 \mathrm{~K}$ and $350 \mathrm{~K}$ respectively (Hasegawa et al. 1992). Katz et al. (1999) have now obtained new values for these numbers by studying molecular hydrogen formation on assorted substances. They obtain 
the following results: for olivine, $E_{\mathrm{b}}=290 \mathrm{~K}, E_{\mathrm{D}}=372 \mathrm{~K}$, and for amorphous carbon, $E_{\mathrm{b}}=511 \mathrm{~K}, E_{\mathrm{D}}=658 \mathrm{~K}$. If we ignore the amorphous carbon results since binding energies are supposed to be abnormally strong on carbon surfaces, we are left with the olivine results, which show that $E_{\mathrm{b}}$ is far higher than our previous estimate. To get some idea what would happen to models if energies against diffusion are generally higher than we have estimated, we have run the simple model of Figure 2 using both modified rate and Monte Carlo approaches with $E_{\mathrm{b}}$ equal to $2 / 3$ of $E_{\mathrm{D}}$. The slowing down of the diffusion rates of $\mathrm{H}, \mathrm{O}$, and $\mathrm{CO}$ changes the chemistry without ruining the agreement between the two approaches. The needed modifications to the rates are minimal in this case. Water is still dominant at most temperatures, but virtually no hydrogenation to form formaldehyde and methanol is detected and molecular oxygen becomes prominent at temperatures above $16 \mathrm{~K}$. If one chooses different values of the gas-phase abundances, the diffusion chemistry can begin to become inoperative. For example, if the following abundances, based on high density steady-state results of Lee et al. (1996) are used $-n_{\mathrm{H}}=1.1 \mathrm{~cm}^{-3}, n_{\mathrm{O}}=7.0 \mathrm{~cm}^{-3}$ and $n_{\mathrm{CO}}=7.5$ $\mathrm{cm}^{-3}$ - up to $30 \%$ of the grain at low temperature is taken up by slow moving $\mathrm{O}$ atoms. In such a situation, both the rate equation and Monte Carlo methods fail (although they fail in different manners) since neither takes account of Eley-Rideal chemistry.

\section{Photochemistry}

Up to now, we have been discussing thermal diffusive chemistry. Laboratory workers in Leiden and elsewhere (Schutte 1999) have shown that surface chemistry on cold objects can be strongly influenced by both photon and particle bombardment. Ruffle \& Herbst (1999, in progress) are attempting to model the photochemistry to be expected in assorted interstellar sources. The basic approach is simple, perhaps even simplistic in nature: since the binding energy of molecules to grains is normally small, the photodissociation and photoionization rates are assumed to be roughly equal to those of gas-phase species. For example, we take the cross section as a function of wavelength for the photodissociation channel of gas-phase $\mathrm{CO}_{2}$ :

$$
\mathrm{CO}_{2}+\mathrm{h} \nu \longrightarrow \mathrm{CO}+\mathrm{O}
$$

to pertain to adsorbed $\mathrm{CO}_{2}$ as well. We are thus able to include rate coefficients for photo-processes of surface species involving both external photons and cosmic ray-induced photons into our gas-grain models. The products of photo-induced reactions are assumed not to subsequently desorb (although experimental evidence on this point is mixed) except for positive ions, which are assumed to desorb after dissociative recombination with electrons (Aikawa \& Herbst 1999). The rate equation method is used for the diffusive chemistry including photons, but the diffusion rate of $\mathrm{H}$ is set at the low value of Katz et al. (1999) because, even if this value is not appropriate for most interstellar grains, slowing the sweeping rate of $\mathrm{H}$ is the most important semi-empirical modification discussed by Caselli et al. (1998).

Preliminary results show that even under typical dense cloud conditions, photochemistry can modify the results of gas-grain models. The most important 
processes appear to break bonds of non-hydrogen-containing molecules such as $\mathrm{CO}$ and $\mathrm{CO}_{2}$. For example, the breaking of the $\mathrm{CO}$ molecule into $\mathrm{C}$ and $\mathrm{O}$ can lead to subsequent hydrogenation to form methane and water, whereas the breaking of methane into radicals and hydrogen is easily reversed.

\section{The Future}

The chemistry occurring on interstellar grains produces both granular and selected gas-phase molecules in interstellar clouds both in star-forming regions and in the ambient medium. Gas-grain chemical models of interstellar clouds are beset by a number of difficulties reflecting uncertainties in surface chemistry, in the chemical and topological nature of the surfaces present in the interstellar medium, and in the mathematical techniques needed to model the chemistry occurring on small particles. Regarding the mathematical difficulties, there are three approaches to consider in future work:

1. Complete Monte Carlo. In this approach, both gas-phase and grain chemistry can be done via the stochastic approach. Unfortunately, nobody has yet managed to accomplish this, and much computer time, probably on a massively parallel machine, will be needed. Charnley (1998) has used a stochastic approach for gas-phase reactions and Tielens \& Hagen (1982) and we have used a simpler Monte Carlo approach for granular chemistry.

2. Mixed rates/Monte Carlo. In this approach, the gas-phase chemistry is treated with rate equations and a Monte Carlo approach is used for the grains. This approach appears to be theoretically possible and should be computationally faster than a complete Monte Carlo approach, but we know of no investigators who have coded this method.

3. Modified rates. The approach of Caselli et al. (1998), Shalabiea et al. (1998), and Caselli \& Herbst (in progress), this method uses rate equations for both gas and grain, with suitable modifications to the grain rate equations. It is practical and certainly achievable with a minimum of time on a supercomputer, but there is no current manner in which one can prove it to be correct in all situations since the semi-empirical grain chemistry must be tested against Monte Carlo approaches.

If one wishes to study photochemistry as well as thermal diffusive chemistry, the most practical method is the modified rates approach although methods based on the other treatments are not outside the realm of possiblity. EleyRideal chemistry, when appropriate, should be treatable via all approaches.

Improvements in mathematical treatments of grain chemistry must go hand in hand with laboratory and theoretical research on the surface chemistry of small particles so that astrochemists can include more precise and correct information in their models. Although the difficulties involved in producing accurate gas-grain chemical models seem large, research in this area remains important to an understanding of interstellar molecules and the physical conditions in which they reside. 
Acknowledgments. The astrochemistry program at Ohio State is supported by the National Science Foundation. Much of the work reported here was done by my collaborator Paola Caselli and my post-doctoral associate Deborah Ruffle.

\section{References}

Aikawa, Y. \& Herbst, E. 1999, ApJ, 526, 314

Allen, M. \& Robinson, G.W. 1977, ApJ, 212, 396

Bergin, E.A., Neufeld, D.A., \& Melnick, G.J. 1999, ApJ, 510, L145

Blake, G.A., Sutton, E.C., Masson, C.R., \& Phillips, T.G. 1987, ApJ, 315, 621

Brown, P.D., Charnley, S.B., \& Millar, T.J. 1988, MNRAS, 231, 409

Brown, P.D. \& Millar, T.J. 1989a, MNRAS, 237, 661

1989b, MNRAS, 240P, 25

Caselli, P., Hasegawa, T.I., \& Herbst, E. 1993, ApJ, 408, 548 1998, ApJ, 495, 309

Charnley, S.B. 1997, in Astrochemical and Biochemical Origins and the Search for Life, eds. S. Bowyer \& D. Wertheimer (Bologna: Editrice Compositori), 89 1998, ApJ, 509, L121

Charnley, S.B., Kress, M.E., Tielens, A.G.G.M., \& Millar, T.J. 1995, ApJ, 448, 232

Charnley, S.B., Tielens, A.G.G.M., \& Millar, T.J. 1992, ApJ, 399, L71

Charnley, S.B., Tielens, A.G.G.M., \& Rodgers, S.D. 1997, ApJ, 482, L203

d'Hendecourt, L.B., Allamandola, L.J., \& Greenberg, J.M. 1985, A\&A, 152, 130 Hasegawa, T.I. \& Herbst, E. 1993a, MNRAS, 261, 83 1993b, MNRAS, 263, 589

Hasegawa, T.I., Herbst, E., \& Leung, C.M. 1992, ApJS, 82, 167

Hiroaka, K., et al. 1994, Chem. Phys. Lett, 229, 408

Hiraoka, K., Miyagoshi, T., Takayama, T., Yamamoto, K., \& Kihara, Y. 1998, ApJ, 498, 710

Irvine, W.M., Goldsmith, P.F., \& Hjalmarson, $\AA$. 1987, in Interstellar Processes, eds. D.J. Hollenbach \& H.A. Thronson, Jr. (Dordrecht: Reidel), 561

Katz, N., Furman, I., Biham, O., Pirronello, V., \& Vidali, G. 1999, ApJ, 522, 305

Lee, H.-H., Bettens, R.P. A., \& Herbst, E. 1996, A\&AS, 119, 111

Millar, T.J. \& Hatchell, J. 1998, Faraday Discussions, 109, 15

Millar, T.J., Herbst, E., \& Charnley, S.B. 1991, ApJ, 369, 147

Pickles, J.B. \& Williams, D.A. 1977, Ap\&SS, 52, 443

Schutte, W.A. 1999, in Laboratory Astrophysics \& Space Research, eds. P. Ehrenfreund, K. Krafft, H. Kochan, \& V. Pirronello (Dordrecht: Kluwer), 69

Shalabiea, O.M. \& Greenberg, J.M. 1995, A\&A, 296, 779

Shalabiea, O.M., Caselli, P., \& Herbst, E. 1998, ApJ, 502, 652

Snyder, L.E., Kuan, Y.-J., \& Miao, Y. 1994, in The Structure and Content of Molecular Clouds, eds. T.L. Wilson \& K.J. Johnston (New York: SpringerVerlag), 187

Terzieva, R. \& Herbst, E. 1998, ApJ, 501, 207

Tielens, A.G.G.M. 1993, in Dust and Chemistry in Astronomy, eds. T.J. Millar \& D.A. Williams (London: IOP Publishing), 103 
Tielens, A.G.G.M. \& Allamandola, L.J. 1987, in Interstellar Processes, eds. D.J. Hollenbach \& H.A. Thronson (Dordrecht: Reidel), 397

Tielens, A.G.G.M. \& Hagen, W. 1982, A\&A, 114, 245

Turner, B.E. 1998, ApJ, 501, 731

Viti, S. \& Wiliams, D.A. 1999, MNRAS, 305, 755

Wagenblast, R., Williams, D.A., Millar, T.J., \& Nejad, L.A.M. 1993, MNRAS, 260,420

Watson, W.D. \& Salpeter, E.E. 1972, ApJ, 175, 659

Willacy, K., Klahr, H.H., Millar, T.J., \& Henning, Th. 1998, A\&A, 338, 995

Williams, D.A. 1993, in Dust and Chemistry in Astronomy, eds. T.J. Millar \&

D.A. Williams (London: IOP Publishing), 143 1998, Faraday Discussions, 109, 1

Wright, M., Sandell, G., Wilner, C.J., \& Plambeck, R.L. 1992, ApJ, 393, 225

Whittet, D.C.B. 1993, in Dust and Chemistry in Astronomy, eds. T.J. Millar \&

D.A. Williams (London: IOP Publishing), 9

\section{Discussion}

S. Charnley: I have 3 comments. (i) The Tielens-Hagen model is not a true Monte Carlo simulation of chemical reactions. For example, the reaction times are not randomly sampled from the correct (exponential) probability distribution. (ii) As one has to implicitly take averages (i.e. number densities) in forming the system of gas phase differential equations, there will be normalization problems if one then attempts to weld such a model with a stochastic treatment of the surface kinetics. (iii) A full stochastic simulation of interstellar gas - grain chemistry requires one to resolve one 'grain' - this sets a lower limit of about $10^{12}$ on the number of gas phase particles required in the calculation.

J. Rawlings: Theoretically, the surface mobility of $\mathrm{H}$ dominates the surface chemistry. How can the recent observations of high $\mathrm{CO}_{2}: \mathrm{CH}_{4}$ ratios and significant abundances of species such as $\mathrm{HCOOH}$ and OCS (in the solid state) towards protostars be reconciled with the models?

E. Herbst: Hydrogenated molecules are not dominant on grain mantles if (a) the gas-phase composition is low in $\mathrm{H}$, (b) the temperature is high enough that $\mathrm{H}$ evaporates before reacting.

$J . M$. Greenberg: Do any of your suggested desorption mechanisms allow sufficient gas-phase molecules beyond $10^{6}$ yrs in a region of $\mathrm{n}_{\mathrm{H}_{2}}=10^{4} \mathrm{~cm}^{-3}$ ?

E. Herbst: In our previous models, we consider cosmic ray - induced desorption only. This desorption mechanism is generally 'inefficient' past $10^{6} \mathrm{yr}$. 


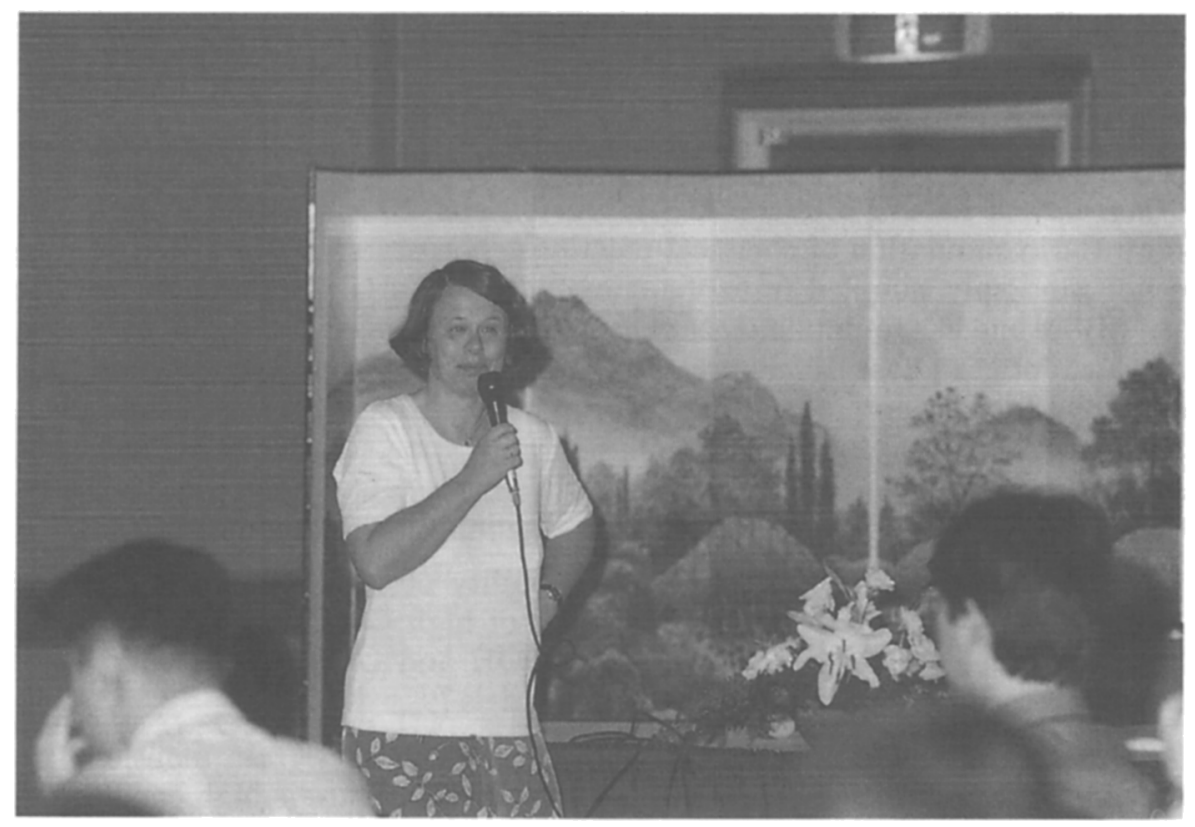

